International Journal of Electrical Engineering and Technology (IJEET)

Volume 11, Issue 4, June 2020, pp. 290-299, Article ID: IJEET_11_04_033

Available online at https://iaeme.com/Home/issue/IJEET? Volume $=11 \&$ Issue $=4$

ISSN Print: 0976-6545 and ISSN Online: 0976-6553

DOI: https://doi.org/10.34218/IJEET.11.4.2020.033

(C) IAEME Publication

Scopus Indexed

\title{
COMPUTATIONALLY EFFICIENT ROBUST MODEL PREDICTIVE CONTROL OF MAGNETIC SUSPENSION SYSTEMS
}

\author{
Ajay Gautam \\ School of Electrical, Electronics and Communication Engineering, \\ Korea University of Technology and Education, Cheonan, South Korea \\ agautam@koreatech.ac.kr
}

\begin{abstract}
Robust model predictive control (MPC) of a general magnetic suspension system (MSS) is analyzed. Tight positioning requirements in the presence of constraints makes MPC a promising control technique for this system. While inherent robustness of the MPC approach may be able to handle some amount of model nonlinearities and external disturbances, we consider a robust approach that systematically incorporates the effects of uncertainties and disturbances in the linearized dynamics model and explore its applicability for a range of operating points or reference inputs of the MSS. Simulation results illustrate the effectiveness and the computational simplicity of the robust MPC approach.
\end{abstract}

Key words: Magnetic suspension system (MSS), model predictive control (MPC), robust MPC.

Cite this Article: Ajay Gautam, Computationally Efficient Robust Model Predictive Control of Magnetic Suspension Systems. International Journal of Electrical Engineering and Technology, 11(4), 2020, pp. 290-299.

https://iaeme.com/Home/issue/IJEET?Volume $=11 \&$ Issue $=4$

\section{INTRODUCTION}

Magnetic suspension systems (MSSs) have been widely and increasingly adopted in industrial applications in the last few decades. While magnetic levitation has been instrumental in the realization of high-speed trains, thus bringing about a paradigm shift in rail transportation [1], contactless bearings in the form of active magnetic bearings [2] have found applications in a variety of areas such as robotics, energy storage systems and artificial heart pumps to name a few [3-5]. Significant advantages of the contactless operation or actuation offered by MSSs include reduced wear due to friction, reduced power losses, noise-free operation, enabling of high-speed motion and the possibility of advanced monitoring and control.

While MSSs offer great advantages, there are significant challenges in their design and operation, including in the areas of physical design, sensing and, particularly, control, since the positioning requirements are usually strict, and the open-loop plant is unstable. Over the 
years, researchers have explored several control techniques for the purpose, including feedback linearization, linear-quadratic, adaptive, sliding mode, and various robust approaches such as $H_{\infty}$ and $\mu$-synthesis [6-12].

With its systematic constraint-handling capacity, model predictive control (MPC) has the potential to meet the tight control specifications for MSSs. However, used primarily for slow processes [13], MPC has not been enthusiastically considered for these systems which are typically characterized by their fast and open-loop-unstable dynamics. Few investigations into the effectiveness of the predictive approach for these systems have explored various forms of MPC including the standard MPC [14], linear-matrix-inequality (LMI)-based MPC [15], nonlinear MPC [16] and so on and they have illustrated a successful constraint-handling by the proposed schemes. However, guaranteeing robustness against uncertainties and disturbances is a critical requirement in the control of MSSs and robust forms of MPC are usually more complex and computationally intensive. In the recent years, computationally efficient forms of robust and nonlinear MPCs have been explored [17-18] and they can be expected to offer better control performances with reasonably simple online computations.

In this paper, we investigate the control of a general MSS with a form of computationally efficient robust MPC that handles model uncertainties and additive disturbances and also offers an $H_{\infty}$-like performance on disturbance attenuation. We model the MSS dynamics for a range of operating points in the form of linear polytopic dynamics with an additive disturbance input representing load and other disturbances and unmodeled nonlinear effects. Inspired by the min-max-cost-based approach explored in [17], we seek to use a dynamic controller whose dynamics are chosen at the design stage to offer a desired level of disturbance attenuation but whose state is optimized online to ensure the satisfaction of constraints and enhance the optimality of the controller. The effectiveness of the chosen approach for this system is illustrated with numerical simulations.

\section{SYSTEM DESCRIPTION}

We consider a general description of a basic magnetic suspension system. As shown in Fig. 1, such a system has 3 subsystems - an object made of some magnetic material that is to be suspended, an electromagnet together with the associated controller and current drive and a combination of light source and sensor used to optically measure the vertical position of the object.

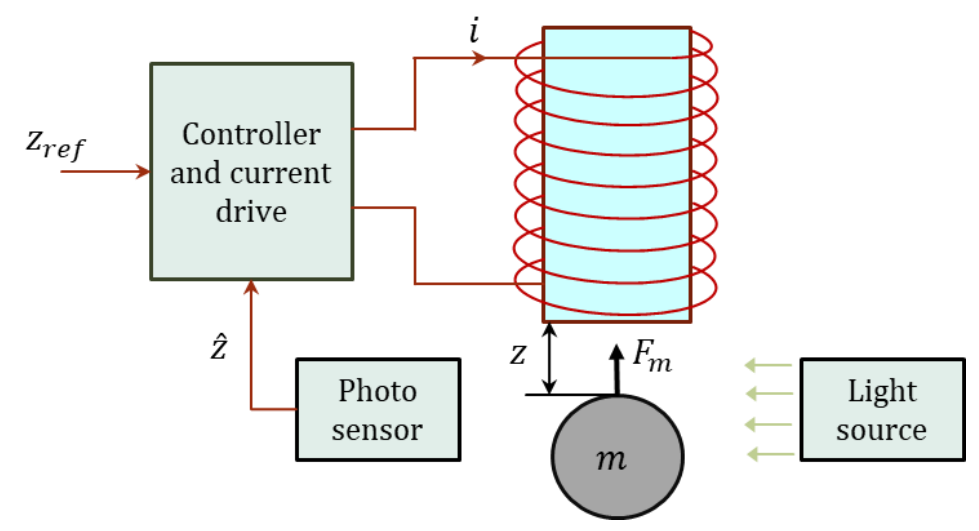

Figure 1 Components of a general magnetic suspension system

Denoting by $z$ the downward vertical position of the object (with $z=0$ representing the position when it is touching the coil), the dynamics of the magnetic object are given by:

$$
m \ddot{z}=-k \dot{z}+m g+F_{m}(z, i)+F_{d}
$$


where $m$ is mass of the object, $k$ is the coefficient of viscous friction, $g$ is the gravitational acceleration, $i$ is the current in the coil and $F_{m}(z, i)$ is electromagnetic force, given by:

$$
F_{m}(z, i)=-\frac{L_{0} a i^{2}}{(a+z)^{2}}
$$

where $L_{0}$ and $a$ are positive constants, and $F_{d}$ is an external additive disturbance force $[19,14]$. The current in the coil is usually driven by a voltage source and there is a nonlinear differential relationship between the coil current and the voltage. However, here, we consider the current in the coil as the control signal. Denoting $z$ and $\dot{z}$ by $z_{1}$ and $z_{2}$, we can write:

$$
\begin{gathered}
\dot{z}_{1}=z_{2} \\
\dot{z}_{2}=g-\frac{k}{m} \dot{z}-\frac{L_{0} a i^{2}}{m(a+z)^{2}}+\frac{1}{m} F_{d}
\end{gathered}
$$

The operating state-input pairs of the system are of the form $\left(\left(z^{o}, 0\right), i^{o}\right)$ where $i^{o}$ is linearly related with $z^{o}$, i.e., $i^{o}=\sqrt{2 M g / L_{0} a}\left(a+z^{o}\right)$.

Linearizing model (1) about an operating state-input pair $\left(\left(z^{o}, 0\right), i^{o}\right)$, we can obtain the following linear model in terms of variables $x_{1}=z_{1}-z^{o}, x_{2}=z_{2}, u=i-i^{o}$ and $w=F_{d}$ :

$$
\dot{x}=\left[\begin{array}{cc}
0 & 1 \\
\frac{2 g}{a+z^{o}} & -\frac{k}{m}
\end{array}\right] x+\left[\frac{0}{\frac{\sqrt{2 L_{0} a g / m}}{a+z^{o}}}\right] u+\left[\begin{array}{c}
0 \\
1 / m
\end{array}\right] w
$$

The linear model in (2) can be discretized to obtain a discrete-time model:

$$
x_{k+1}=A x_{k}+B u_{k}+D w_{k}
$$

where $k \geq 0$ denotes the time step. Note here that the dimensions of $x_{k}, u_{k}$ and $w_{k}$ are $n_{x}=2, n_{u}=1$ and $n_{w}=1$. Note also that the state components, the input and the disturbance are required or assumed to have their values within some limits: $x_{k} \in \mathbb{X}, u_{k} \in \mathbb{U}$ and $w_{k} \in \mathbb{W}$, where $\mathbb{X}, \mathbb{U}$, and $\mathbb{W}$ are convex and compact sets.

We wish to design a model predictive controller that is applicable for a range of operating points and satisfies a relevant $H_{\infty}$ criterion that limits the gain from the disturbances to the output. To make the design applicable for a range of operating points, we consider a convex set of system matrices in model (3), described in the form of a convex hull specified by a set of vertex matrices:

$$
S_{A B D}=\left\{\left[\begin{array}{lll}
A & B & D
\end{array}\right] \mid\left[\begin{array}{lll}
A & B & D
\end{array}\right]=\sum_{i}^{L} \lambda_{i}\left[A_{i} B_{i} D_{i}\right], \sum_{i}^{L} \lambda_{i}=1, \lambda_{i} \geq 0, i=1, \ldots, L\right\}
$$

Some care is needed when finding the vertex matrices $\left[\begin{array}{lll}A_{i} & B_{i} & D_{i}\end{array}\right], i=1, \ldots, L$ for the set of discrete-time system matrices in (4) and this will be discussed in Section 4.

\section{ROBUST MODEL PREDICTIVE CONTROL}

Model predictive control is based on an online optimization, at each time step, $k$, of a sequence of future control inputs in order to obtain an input sequence that minimizes a suitably defined cost function $J(k)$ based on the control objective. From the optimized sequence of control inputs, the input meant for the current time step $k$ is applied to the system and, at the next time step, the optimization process is repeated again. 


\subsection{Cost Function}

The MPC cost function typically is a quadratic function, and, in the standard form, it is evaluated explicitly for a finite horizon of $N$ time steps:

$$
J_{F H}(k)=\sum_{i=0}^{N-1}\left\{x_{k+i+1 \mid k}^{T} Q x_{k+i+1 \mid k}+u_{k+i \mid k}^{T} R u_{k+i \mid k}\right\}
$$

Here, $P, Q$ are positive semidefinite matrices and $R$ is a positive definite matrix. The quantities $x_{k+i \mid k} u_{k+i \mid k}$ denote predicted values of the state and the input of the future time step $k+i$ based on the computation made in the current time step $k$.

Since we have a system model with unknown disturbances, the cost function in (5) cannot be evaluated exactly, and to be able to guarantee stability, we need to consider additional cost beyond the finite horizon. So, we consider the following worst-case infinite-horizon cost:

$$
J(k)=\max _{\substack{A B \\ B \\ w(k) \in \mathbb{W}}} \sum_{i=0}^{N}\left\{x_{k+i \mid k}^{T} Q x_{k+i \mid k}+u_{k+i \mid k}^{T} R u_{k+i \mid k}-\gamma^{2} w_{k+i \mid k}^{T} w_{k+i \mid k}\right\}
$$

evaluated for $N \rightarrow \infty$, where $\gamma$ is the disturbance attenuation parameter.

\subsection{Control input Parametrization and Offline Computations}

It is not possible to optimize the control input sequence for the infinite horizon. We, therefore, follow the method explored in [17], and parameterize the predicted control inputs as:

$$
u_{k+i \mid k}=K x_{k+i \mid k}+H \xi_{k+i \mid k}
$$

where $K$ is a stabilizing feedback gain (stabilizing for the set $S_{A B D}$ of system matrices) and $\xi_{k+i \mid k}, i \geq 0$ are predicted future states of a stable controller system described by:

$$
\xi_{k+1}=G \xi_{k}
$$

We can allow matrix $G$ to vary in a convex set in the same way as matrices $A, B$, and $D$. Here, we optimize the vector $\xi_{k}$ online but we choose matrices $H$ and $G$ (or the vertices of the set in which it lies) offline. An LMI-based procedure to choose these controller matrices in order to improve the region of attraction of the resulting controller is described in [17]. Alternatively, we can make some straightforward choices:

$$
G=\left[\begin{array}{ccccc}
0 & I_{n_{u}} & \cdots & 0 & 0 \\
0 & 0 & \cdots & 0 & 0 \\
\vdots & \vdots & \ddots & \vdots & \vdots \\
0 & 0 & 0 & 0 & I_{n_{u}} \\
0 & 0 & 0 & 0 & 0
\end{array}\right], \quad H=\left[\begin{array}{llll}
I_{n_{u}} & 0 & \cdots & 0
\end{array}\right]
$$

so that we can freely optimize the input sequence for a number of future times steps. Combining the system dynamics (3) with the dynamics of the controller ( $7 b$ ), we get an augmented system:

$$
\zeta_{k+1}=\mathcal{A} \zeta_{k}+\mathcal{D} w_{k}
$$

where

$$
\zeta_{k}=\left[\begin{array}{l}
x_{k} \\
\xi_{k}
\end{array}\right], \quad \mathcal{A}=\left[\begin{array}{cc}
A+B K & B H \\
0 & G
\end{array}\right], \quad \mathcal{D}=\left[\begin{array}{l}
D \\
0
\end{array}\right]
$$

Considering the fact that $\left[\begin{array}{lll}A & B & C\end{array}\right] \in S_{A B D}$, matrices $\mathcal{A}$ and $\mathcal{D}$ also lie in a convex set, say, defined by vertex matrices $\left[\mathcal{A}_{i} \mathcal{D}_{i}\right], i=1,2, \ldots, L$. The augmented system is stable. We can compute the region of attraction of this augmented closed-loop system. In particular, we compute the largest invariant set $\mathcal{Z}$ such that all state and input constraints are satisfied for all $\left[\begin{array}{l}x_{k} \\ \xi_{k}\end{array}\right] \in \mathcal{Z}$, i.e., a set $Z$ that satisfies: 


$$
\begin{aligned}
& \mathcal{A}_{i} Z \oplus \mathcal{D}_{i} \mathbb{W} \subseteq \mathcal{Z}, i=1, \ldots, L \\
& \chi=\operatorname{Proj}_{x}(\mathcal{Z})=\left[\begin{array}{ll}
I_{n_{x}} & 0
\end{array}\right] \mathcal{Z} \subseteq \mathbb{X}, \quad\left[\begin{array}{ll}
K & H
\end{array}\right] \mathcal{Z} \subseteq \mathbb{U}
\end{aligned}
$$

where $\oplus$ denotes set addition. Such a set is a polyhedral set of the form $\{\zeta \mid M \zeta \leq 1\}$, where 1 denotes a vector of all ones. Iterative algorithms computing a close approximation of such an invariant set are available (see, e.g., [20]).

\subsection{Online MPC Computations}

We need to compute the controller state $\xi_{k}$ online by minimizing the cost function. Under the input description in (7), the worst-case cost $J(k)$ in (6) is upper bounded by $\zeta_{k}^{T} P \zeta_{k}$ where $P$ is a positive definite matrix satisfying the LMI condition [17]

$$
\left[\begin{array}{cccc}
P & \mathcal{A}_{i}^{T} P & C_{\zeta}^{T} & 0 \\
P \mathcal{A}_{i} & P & 0 & P \mathcal{D}_{i} \\
C_{\zeta} & 0 & I & 0 \\
0 & \mathcal{D}_{i}^{T} P & 0 & \gamma^{2} I
\end{array}\right] \geq 0, \quad i=1,2, \ldots, L, \quad C_{\zeta}=\left[\begin{array}{cc}
Q^{\frac{1}{2}} & 0 \\
R^{\frac{1}{2}} K & R^{\frac{1}{2}} H
\end{array}\right]
$$

This condition ensures that for the augmented system (9), we have, $\zeta_{k+1}^{T} P \zeta_{k+1}-\zeta_{k}^{T} P \zeta_{k} \leq$ $-\zeta_{k}^{T} C_{\zeta}^{T} C_{\zeta} \zeta_{k}+\gamma^{2} w_{k}^{T} w_{k}$, and therefore, for all $N \geq 0$,

$$
\zeta_{0}^{T} P \zeta_{0} \geq \sum_{k=0}^{N}\left\{\zeta_{k}^{T} C_{\zeta}^{T} C_{\zeta} \zeta_{k}-\gamma^{2} w_{k}^{T} w_{k}\right\}
$$

where $\zeta_{k}^{T} C_{\zeta}^{T} C_{\zeta} \zeta_{k}$ is equal to the stage cost $x_{k}^{T} Q x_{k}+u_{k}^{T} R u_{k}$.

We compute a positive definite cost matrix $P$ that satisfies (11) and is also 'small' in some sense, e.g., has the minimum trace or determinant. Having obtained the invariant set $Z$ and the cost matrix $P$ offline, the MPC optimization problem at time $\mathrm{k}$ can be stated as:

$$
\begin{array}{ll}
\underset{\xi_{k}}{\operatorname{minimize}} & {\left[\begin{array}{ll}
x_{k}^{T} & \xi_{k}^{T}
\end{array}\right] P\left[\begin{array}{l}
x_{k} \\
\xi_{k}
\end{array}\right]} \\
\text { subject to } & {\left[\begin{array}{l}
x_{k} \\
\xi_{k}
\end{array}\right] \in Z}
\end{array}
$$

Algorithm 1 - Online MPC algorithm: At each time step $k$ :

- Measure or estimate the state of the system $x(k)$

- Solve the optimization problem in (12) to obtain an optimal $\xi_{k}^{*}$

- Apply the control input $u_{k}^{*}=K x_{k}+H \xi_{k}^{*}$ to the plant

Theorem 1: The MPC algorithm of Algorithm 1 ensures that the MPC optimization problem is feasible at all times, the state and input constraints are always satisfied and that the controlled system satisfies the condition:

$$
\sum_{k=0}^{N}\left\{x_{k}^{T} Q x_{k}+u_{k}^{* T} R u_{k}^{*}\right\} \leq \zeta_{0}^{T} P \zeta_{0}+\gamma^{2} \sum_{k=0}^{N} w_{k}^{T} w_{k}
$$

for all possible values of $x_{0} \in \mathcal{X},\left[\begin{array}{lll}A & B & D\end{array}\right] \in S_{A B D}$ and $w_{k} \in \mathbb{W}, k \geq 0$.

This result follows directly from the properties in (10) of the set $Z$ and the properties of the cost matrix P. See [17] for details. 


\section{ROBUST MPC OF A MAGNETIC SUSPENSION SYSTEM}

\subsection{System Parameters}

We consider a specific example of the magnetic suspension system with the following details (see, e.g., [19, Chap.12]):

$$
\begin{aligned}
& m=0.1 \mathrm{~kg}, \quad g=9.81 \mathrm{~ms}^{-2}, \quad k=0.001 \mathrm{Nsm}^{-1}, \\
& a=0.05 \mathrm{~m}, \quad L_{0}=0.01 \mathrm{H}
\end{aligned}
$$

The nominal operating point corresponds to $z^{o}=0.05$ but we consider a range of operating points for $z^{o} \in[0.01,09] \mathrm{m}$. Note that the system matrices in the linearized model (2) depend linearly on the parameter $1 /\left(a+z^{o}\right)$. The corresponding matrices in the discretized model (3), which we have obtained for a sampling time of $T=0.02 \mathrm{~s}$, have elements that depend on this parameter in a nonlinear way. But an analysis of the relationship between various elements of the discrete-time system matrices $A, B$ and $D$ shows that they all are nearly linearly related to each other for the example here. Therefore, we choose the discrete time system matrices corresponding to $z^{o}=0.01 \mathrm{~m}$ and $z^{0}=0.09 \mathrm{~m}$ as the vertices of the set $S_{A B D}$.

\subsection{Controller details and Offline Computations}

We consider $Q=\left[\begin{array}{cc}1 & 0 \\ 0 & 0.1\end{array}\right]$ and $R=0.01$ in the cost function and assume the following constraints: $z \in[0,0.1] \mathrm{m}, \dot{z} \in[-0.5,0.5] \mathrm{m} / \mathrm{s}, i \in[0,12] \mathrm{A}$ and $F_{d} \in[-0.2,0.2] \mathrm{N}$.

Next, we obtain a feedback gain $K$ that is $H_{\infty}$ optimal in the sense of minimizing the gain from $w_{k}$ to $y_{k}=\left(Q^{1 / 2} x_{k}, R^{1 / 2} u_{k}\right)$ for all system matrices in $S_{A B D}$ and consider the controller matrices as in (8) with $n_{\xi}=4$. Since the MPC formulation is based on the state and input deviations $x=\left(z_{1}, z_{2}\right)-\left(z^{o}, 0\right)$ and $u=i-i^{o}$, the constraints on $x$ and $u$ depend on the choice of the operating point. So, while determining the invariant set $Z$, we find its extension $Z^{e}$ for the vector $\zeta_{k}^{e}=\left(x_{k}, \Delta z^{o}, \xi_{k}\right)$ where $\Delta z^{o}$ is the deviation of $z^{o}$ from its nominal values of $0.05 \mathrm{~m}$. Clearly $\Delta z^{o} \in[-0.04,0.04]$. The projections of the set $Z^{e}$ obtained under the assumed constraints are shown in Fig. 2.
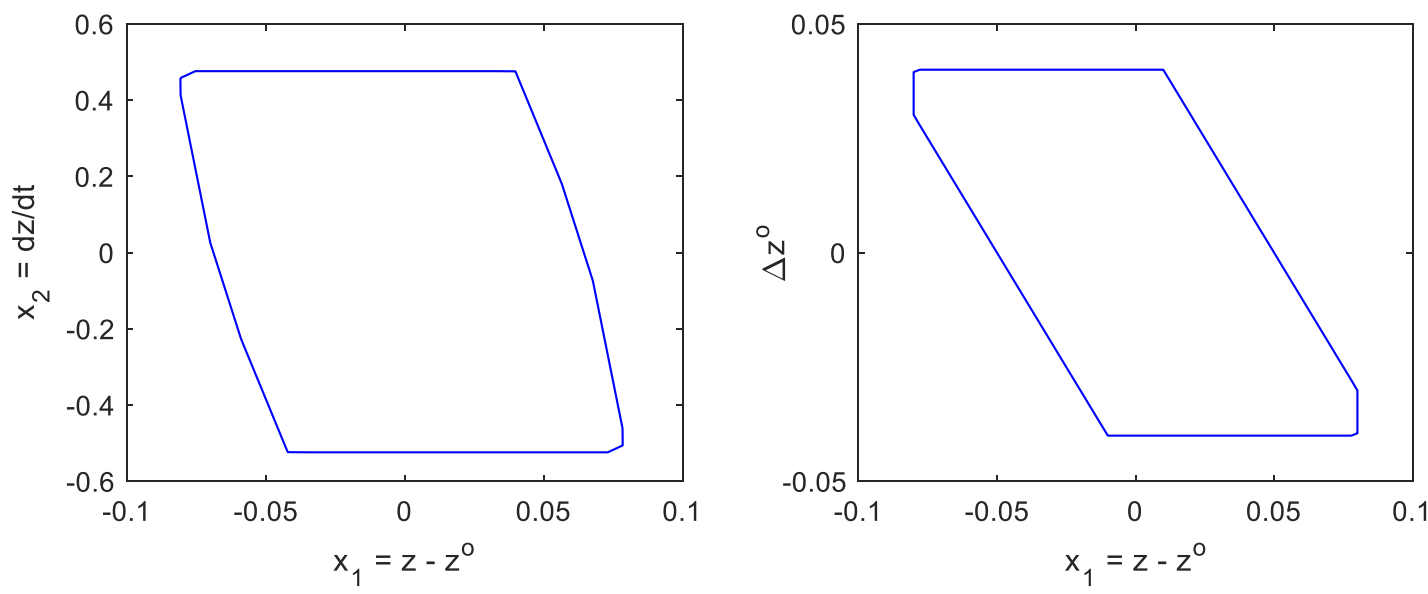

Figure 2 Projections of the set $Z^{e}$ on $x_{1}-x_{2}$ and $x_{1}-\Delta z^{o}$ subspaces

We obtain cost matrix $P$, by minimizing the trace of $P$ under the condition in (11) using the value of $\gamma$ obtained for the gain matrix $K$ (which is just below 0.6). 


\subsection{Simulation Results}

We consider a control scenario in which $z(0)=0.092 \mathrm{~m}$ and $\dot{z}(0)=0$. The operating point is changed a few times. We choose $z^{o}=0.03 \mathrm{~m}$ for $t=0$ to $1 \mathrm{~s}, z^{o}=0.01 \mathrm{~m}$ for $t=1 \mathrm{~s}$ to $2 \mathrm{~s}$ and $z^{o}=0.07 \mathrm{~m}$ for $t=2 \mathrm{~s}$ to $3 \mathrm{~s}$. Fig. 3(a) shows the response of the controlled system under the MPC scheme of Algorithm 1. We can see that all constraints are satisfied at all times and the system is able to successfully track the desired operating points. Fig. 3(b) shows the response of the controlled system under the static feedback input with gain $K$. While constraints are not respected, this controller leads to a situation where it is unable to track the new operating point after $2 \mathrm{~s}$. In both the cases, the disturbance input used comprised a uniformly distributed random signal within $\pm 0.1 \mathrm{~N}$ together with sporadic impulses of magnitude up to $0.2 \mathrm{~N}$.

Next, we consider the case of a gradual change in the operating point. Fig. 4(a) shows the position response (blue curve) when we make linear changes in $z^{0}$ as indicated by the red line. The corresponding control signal is shown in Fig. 4(b). There is some deviation in the tracking of the ramp reference signal and this may be reduced by introducing an integrator element which can also be useful in the case of constant disturbances existing for a long time.
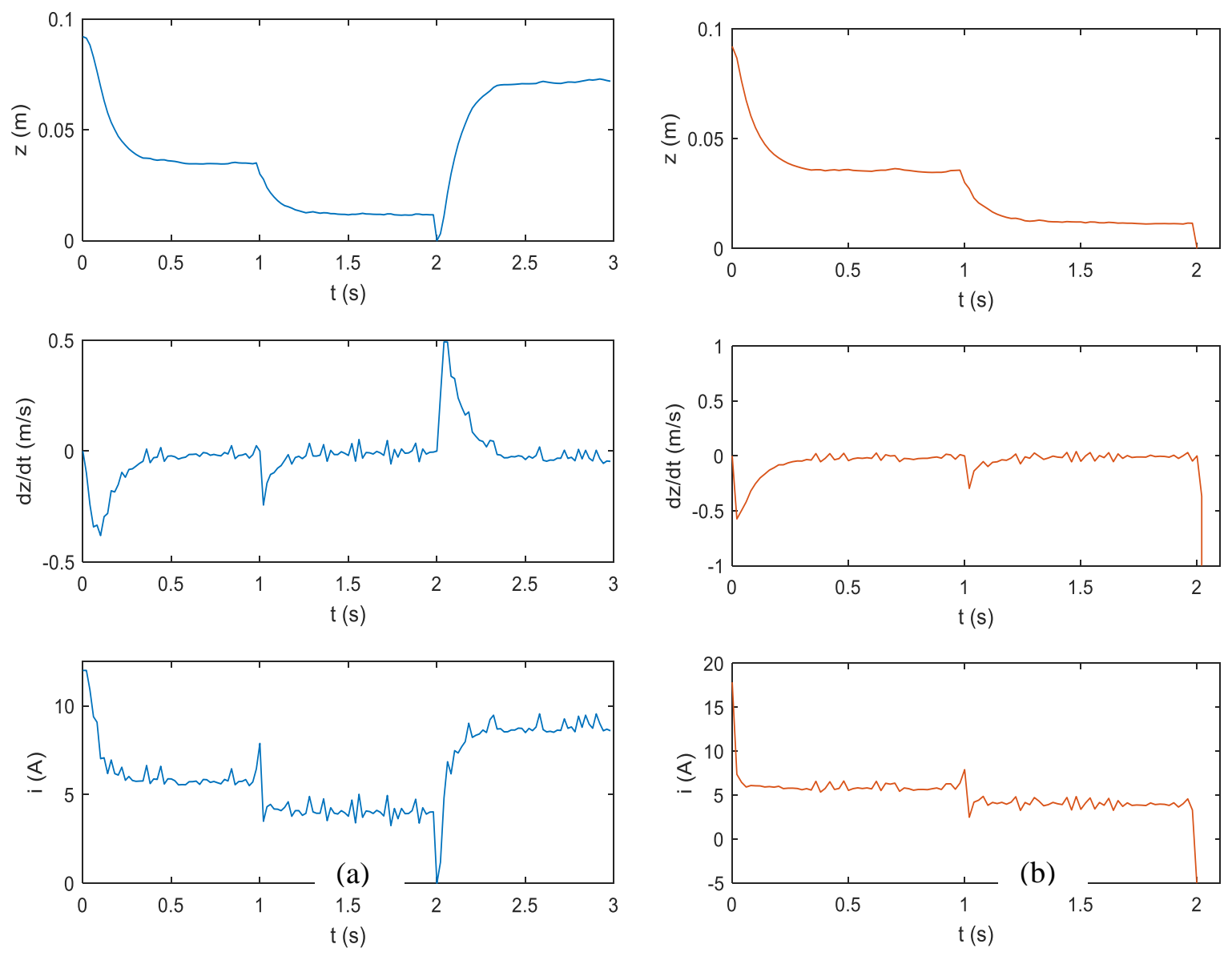

Figure 3 Vertical position and velocity of the magnetic object and the coil current under robust MPC algorithm (a) and under static-state feedback (b) 


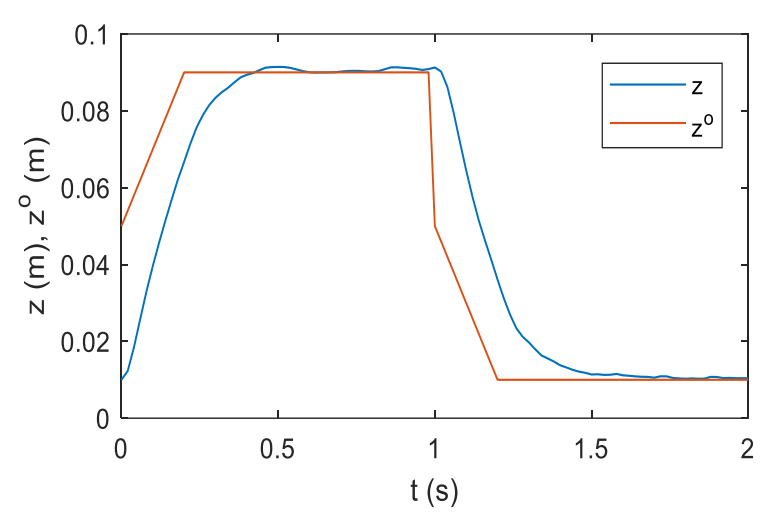

(a)

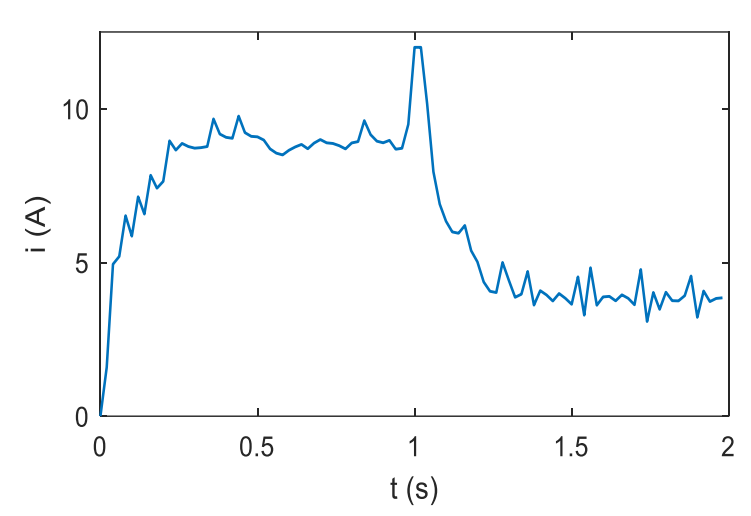

(b)

Figure 4 Position response of the controlled system for a piecewise affine reference position (a), and the corresponding control input (b)

Finally, note that we have assumed the full knowledge of the state of the system, i.e., the position and velocity of the magnetic object. The velocity of the object can also be estimated from the measurements of the position by using an observer. Also, note that the linearized model in (3), does not explicitly incorporate the unmodeled higher order nonlinearities in its uncertainty description. For this reason, there is always a possibility of the MPC problem, that is initially feasible, turning infeasible at a later time step. We considered a set of 200 randomly chosen points from the second set in Fig. 2 for which the MPC problem is feasible with $\dot{z}=0$. It was observed that the problem turned infeasible for $3.5 \%$ of the cases within the first 3 steps whereas for another $1.5 \%$ of the cases infeasibility was observed at a later time step. This problem can be addressed by softening the constraint in case of an infeasible solution.

\subsection{Computational Complexity}

The robust MPC scheme explored in this paper requires the computation of the invariant set $Z$ and the cost matrix $P$ at the design stage which poses no computational difficulties. The online computations include the solution of a quadratic programming problem with $n_{\xi}$ optimization variables and $m$ linear constraints where $m$ is the number of rows of the matrix $M$ defining the set $\mathcal{Z}$. In the problem considered here, we have chosen $n_{\xi}=4$ and $m$ is less than 100. This is a computationally simple problem compared with other alternatives. The standard finite horizon MPC does not explicitly handle the presence of uncertainties or disturbances but still it may require a larger number of optimization variables. For the LMIbased MPC, we need to solve a semidefinite programming problem online and with the nonlinear MPC, the problem is significantly more complex, albeit possibly more optimal in some cases.

\section{CONCLUSION}

The study in this paper has illustrated the effectiveness of the use of a dynamic-controllerbased robust MPC for the control of a magnetic suspension system. The MPC scheme guarantees the satisfaction of system constraints while also guaranteeing of an $H_{\infty}$-like performance criterion for the attenuation of disturbances with a gain parameter that is nearly optimal. Several extensions of the method can be considered, e.g., the incorporation of an estimator to handle cases where the velocity measurement is not available, or to estimate a deterministic external disturbance, the incorporation of an integrator to deal with constant disturbances and so on. 


\section{ACKNOWLEDGEMENT}

This paper was supported by Education and Research promotion program of KOREATECH in 2018.

\section{REFERENCES}

[1] H.W. Lee, K.C. Kim, and J. Lee, (2006) Review of Maglev train technologies, IEEE Transactions on Magnetics, vol. 42, no. 7, pp. 1917-1925.

[2] A. Chiba, T. Fukao, O. Ichikawa, M. Oshima, M. Takemoto, and D.G. Dorrell, (2005) Magnetic Bearings and Bearingless Drives, Elsevier, Amsterdam, The Netherlands.

[3] J. Abbott, E. Diller, and A. Petruska, (2020) Magnetic methods in robotics, Annual Review of Control, Robotics, and Autonomous Systems, vol. 3, pp. 57-90.

[4] C. Zhang and K.J. Tseng, (2007) A novel flywheel energy storage system with partially self-bearing flywheel rotor. IEEE Transactions on Energy Conversion, vol. 22, no. 2, pp. 477-487.

[5] X. Song, A.L. Throckmorton, A. Untaroiu, S. Patel, P.E. Allaire, H.G. Wood, and D.B. Olsen, (2000) Axial flow blood pumps. ASAIO Journal, vol. 49, pp. 355-364.

[6] C.-H. Kim, H. Ahn, J. Lee, and H. Lee, (2017) Linear quadratic servo design for magnetic levitation systems considering disturbance forces from linear synchronous motor, Journal of Electrical Engineering \& Technology, vol. 12, no. 2, pp. 944-949.

[7] M. Maggiore and R. Becerril, (2004) Modelling and control design for a magnetic levitation system, International Journal of Control, Vol. 77, No. 10, pp. 964-977.

[8] Z. Zhang and X. Li, (2018) Real-time adaptive control of a magnetic levitation system with a large range of load disturbance, Sensors, vol. 18, pp. 1-15.

[9] S. Sivrioglu and K. Nonami, (1998) Sliding mode control with time-varying hyperplane for AMB systems. IEEE/ASME Transactions on Mechatronics, vol. 3, pp. 51-59.

[10] K. Nonami, W. He, and H. Nishimura, (1994) Robust control of magnetic levitation systems by means of $\mathrm{H} \infty$ control $/ \mu$-synthesis. JSME International Journal Ser. C: Dynamics, Control, Robotics, Design and Manufacturing, vol. 37, 513-520.

[11] A. Javadi, G. Alizadeh, A. R. Ghiasi, and S. Pezeshki, (2014) Robust control of electromagnetic levitation system, Journal of Control, Automation and Electrical Systems, vol. 25 , no. 5 , pp. $527-536$.

[12] C.-H. Kim, (2017) Robust control of magnetic levitation systems considering disturbance force by LSM propulsion systems, IEEE Transactions on Magnetics, vol.53, no. 11.

[13] J. M. Maciejowski, (2002) Predictive Control with Constraints, Prentice Hall, England.

[14] R.C. Fama et al., (2006) Predictive Control of a Magnetic Levitation System with Explicit Treatment of Operational Constraints, ABCM Symposium Series in Mechatronics, vol.2, pp. 1-8.

[15] M.S. Matos, R.K.H. Galvão, and T. Yoneyama, (2010) Robust model predictive control for a magnetic levitation system employing linear matrix in qualities, ABCM Symposium Series in Mechatronics, vol.4, pp. 147-155.

[16] T. Bächle, S. Hentzelt, and K. Graichen, (2013) Nonlinear model predictive control of a magnetic levitation system, Control Engineering Practice, vol. 21, No.9, pp 1250-1258.

[17] A. Gautam, Y.-C. Chu, and Y. C. Soh, (2012) Optimized dynamic policy for receding horizon control of linear time-varying systems with bounded disturbances, IEEE Transactions on Automatic Control, vol. 57, no. 4, pp. 973-988. 
Computationally Efficient Robust Model Predictive Control of Magnetic Suspension Systems

[18] A. Gautam and Y. C. Soh, (2015) Stabilizing model predictive control using parameterdependent dynamic policy for nonlinear systems modeled with neural networks, Journal of Process Control, vol. 36, pp. 11-21.

[19] H.K. Khalil, (2002) Nonlinear Systems ( ${ }^{\text {rd }}$ edition), Prentice Hall, Upper Saddle River, NJ.

[20] F. Blanchini and S. Miani, (2008) Set-Theoretic Methods in Control, Springer. 